INRA Prod. Anim., 2011, 24 (2), 181-190

\title{
Gérer l'alimentation pour contribuer au bien-être des poulets de chair
}

\author{
M. MAGNIN 1 , I. BOUVAREL 2 \\ ${ }^{1}$ BNA Nutrition Animale, Z.I. de Bellitourne F-53200 Château-Gontier, France \\ 2 ITAVI, Centre INRA de Tours, F-37380 Nouzilly, France \\ Courriel :michel.magnin@bna-na.fr
}

\begin{abstract}
L'évolution de la sélection génétique conduit à produire des poulets à très fort potentiel de croissance avec des effets induits sur l'expression des comportements et les capacités d'adaptation. Peut-on améliorer le bien-être des poulets de chair en jouant sur la conduite alimentaire afin de limiter l'apparition des troubles locomoteurs, digestifs et métaboliques ?
\end{abstract}

Le bien-être d'un animal dit de rente ne peut être respecté que si son environnement et les pratiques d'élevage le mettent à l'abri de la faim, de la soif, de la douleur, des blessures et des maladies, de la peur et du stress, et lui permettent d'exprimer un comportement «normal» pour l'espèce (Manning et al 2007). Ces désordres sont souvent difficiles à appréhender (définition, mesure, échelle, normes) (Arnould 2005, Allain et al 2009) et leur apparition est d'origine multifactorielle. De plus, la sélection des poulets de chair sur la vitesse de croissance, l'efficacité alimentaire et l'engraissement contribue à une évolution du comportement, avec des animaux moins sensibles aux facteurs anorexigéniques (Cassy et al 2004) et moins actifs (Nielsen 2004), complexifiant le problème.

La conduite alimentaire peut être impliquée dans l'apparition de difficultés locomotrices, de troubles digestifs, de dégradation de litière et, par conséquent, de lésions cutanées (pododermatites, brûlures des tarses, ampoules du bréchet) et d'agressions respiratoires, et enfin de la mortalité (arrêt cardiaque, stress thermique, ascites). Elle peut permettre par ailleurs d'enrichir le milieu de vie des volailles dans les élevages en claustration.

La directive 2007/43/CE sur la protection des poulets de chair impose une densité maximale de référence à un instant donné de $33 \mathrm{~kg} / \mathrm{m}^{2}$ mais prévoit des exceptions permettant un chargement supérieur, liées à la maîtrise de signaux d'agression comme la mortalité; l'intensité des pertes d'intégrité cutanée détectées à l'abattage, comme les pododermatites, est également considérée comme un indicateur poten- tiel de bien-être. Notre propos est donc d'envisager différentes approches alimentaires et nutritionnelles de manière à éviter l'apparition de ces désordres.

\section{1 / Un aliment accessible, équilibré et sain}

Un prérequis concernant l'alimentation des volailles est que les animaux reçoivent un aliment accessible, équilibré et sain. Ceci passe par la disponibilité de l'eau et de l'aliment, la qualité physico-chimique et bactériologique de l'eau et l'apport de nutriments essentiels à la vie.

\section{1 / Disponibilité de l'eau et de l'aliment}

Pour assurer une disponibilité acceptable de l'eau et de l'aliment, le nombre de points d'abreuvement et d'alimentation doit être en adéquation avec le nombre de poulets présents, en prenant en compte l'âge des animaux et les conditions d'ambiance. Différents guides d'élevage comme par exemple ceux des sélectionneurs apportent des recommandations à ce sujet. Le débit effectif de l'eau, en particulier pour les dispositifs en pipettes, doit être vérifié fréquemment car potentiellement très variable (Dozier 2003).

De plus, d'après la directive 2007/43/CE, les abreuvoirs doivent être placés et entretenus de façon à réduire au maximum tout déversement accidentel. Les poulets peuvent être alimentés ad libitum ou par repas, les animaux ne pouvant être privés d'aliment plus de douze heures avant l'heure d'abattage prévue.

\section{2 / Qualité de l'eau de boisson}

La composition chimique et les qualités physiques et microbiologiques de l'eau de boisson sont des facteurs importants de variation de la quantité consommée et, par conséquent, de la quantité d'urine et d'eau fécale émises et donc de l'état de la litière (humidité, intensité des fermentations) (Manning et al 2007). Il n'existe pas de normes spécifiques pour l'eau destinée à l'abreuvement des volailles. Néanmoins, différentes recommandations sont proposées (Montiel 2007, ITAVI 2007, Travel et al 2007).

\section{3 / Distribuer un aliment qui répond à des «besoins»}

Les volailles doivent recevoir par ailleurs un aliment apportant les éléments essentiels à la vie, c'est-à-dire une quantité suffisante de macronutriments (protéines, lipides, glucides) apportés par les matières premières et de micronutriments (vitamines, minéraux et oligoéléments) nécessaires pour assurer la couverture de l'ensemble des besoins physiologiques, en évitant toute carence alimentaire visible. L'animal cherche tout d'abord à assurer son équilibre énergétique et adapte son ingestion en fonction des nutriments disponibles. Ceci est réalisé simultanément avec la recherche de l'homéostasie protéique et de l'homéothermie.

Toutefois, la définition de besoins nutritionnels est difficile car liée à l'individu, à un moment donné dans son environnement :

- En conditions de production, des aliments complets sont distribués successivement au cours de la vie des volailles avec une baisse progressive des apports 
en protéines. Cependant, le rapport énergie/protéines n'est approprié qu'à un temps donné et pour des animaux «moyens» sans prendre en compte les différences entre individus en termes de croissance, en particulier entre les mâles et les femelles de même âge (Shariatmadari et Forbes 1993) lorsque les animaux sont élevés ensemble ;

- Théoriquement, pour des conditions de température optimale, le niveau d'ingestion dépend de la valeur énergétique de l'aliment, l'équilibre de la balance énergétique se concrétisant par l'ajustement de l'énergie ingérée à la concentration de l'aliment. Or, le poulet de chair à croissance rapide consomme plus d'énergie avec un aliment à haute concentration énergétique qu'avec un aliment moins concentré en conditions thermiques non limitantes (Leeson et Summers 2000, Quentin et al 2003). En termes de bien-être, se pose la question de savoir s'il faut favoriser le poulet gros mangeur ou le restreindre, et de l'apport énergétique minimum à satisfaire ;

Un autre exemple illustrant la difficulté de fixer des normes alimentaires minimales est celui de l'apport vitaminique. Halley (2007) comparant différentes sources donne des recommandations pour l'aliment démarrage allant de 1500 à $12500 \mathrm{UI} / \mathrm{kg}$ pour la vitamine $\mathrm{A}$, de 200 à $5000 \mathrm{UI} / \mathrm{kg}$ pour la vitamine D3 ;

Pour les oligo-éléments inorganiques, les apports peuvent varier d'un facteur 2 ou 4, sans compter que certaines recommandations incluent des apports en molybdène ou en cobalt et d'autres non. Par ailleurs, certains oligo-éléments pourraient présenter un intérêt notamment en termes d'intégrité et de qualité osseuse (bore, silicium) mais ne sont pas autorisés aujourd'hui par la législation européenne (Nys 2001, OviedoRodon et al 2006).

Ainsi, il n'est pas aisé de définir des «besoins nutritionnels». En pratique, la formulation des aliments repose sur des normes propres aux entreprises, représentant une somme de compromis.

\section{4 / Distribuer un aliment «sain»}

Outre la couverture de ses besoins nutritionnels, le poulet doit recevoir aussi un aliment dont un ou plusieurs composants ne doivent pas provoquer une atteinte directe à son bien-être ou à son intégrité physique, ce que nous appellerons «toxicité» au sens large. Les principaux effets toxiques de différents composants potentiels des aliments sont présentés selon la nature de la toxicité des ingrédients :

\section{a) Toxicité des composants introduits} volontairement dans l'aliment

Les effets des apports élevés en acides aminés soufrés comme la méthionine de synthèse par exemple, comme celui de la L-cystine, peuvent provoquer des baisses de consommation d'aliment dramatiques (Summers 1995, Baker 2008). Les molécules coccidiostatiques ionophores utilisées dans l'aliment pour la prévention des coccidioses ont un coefficient de sécurité, entre les doses efficace et toxique, très faible même chez le poulet de chair, le plus tolérant des volailles.

\section{b) Toxicité due à la nature intrinsèque des matières premières}

Beaucoup de matières premières, en particulier d'origine végétale entrant dans la composition des aliments, contiennent des molécules de nature variée et plus ou moins complexe dénommées «facteurs antinutritionnels» (FAN), venant interagir avec le niveau d'ingestion ou le processus de digestion du poulet (D'Mello 2000). Les composants qui ont un effet dépréciateur sur la consommation de l'aliment sont d'importance toute particulière :

- facteurs antitrypsiques des graines de soja crues ou mal cuites (Esmail 1998);

- tannins condensés du sorgho (Nyamambi et al 2007);

- tannins, vicine, convicine de certaines fèveroles (Métayer et al 2003);

- acide sinapique du colza (Qiao et al 2008) ;

- gossypol du coton (Lordelo et al 2004).

La baisse de digestibilité liée à la présence de ces FAN, porte essentiellement sur les protéines. Ceci peut favoriser le développement de populations microbiennes du tube digestif distal comme des clostridies pathogènes (Drew et al 2004) et contribuer également à l'enrichissement en azote ammoniacal de la litière.

D'autres FAN peuvent augmenter la viscosité intestinale du poulet, baisser la digestibilité des nutriments et augmenter la teneur en eau des fientes, comme les arabinoxylanes et béta-glucanes solubles de céréales (Carré et al 1994), des oligosaccharides présents dans le soja (KarrLilienthal et al 2005, de Coca-Sinova et al 2010) ou les mucilages des graines de lin (Rebolé et al 2002).

Afin de prévenir ou limiter les effets des FAN, il est conseillé de proscrire ou limiter l'usage des matières premières riches en FAN surtout dans l'aliment du jeune poulet, ou bien d'appliquer des traitements technologiques (cf. $\S 2.1$ ). c) Toxicité due à la qualité dégradée des matières premières

Des matières premières peuvent voir leur qualité dégradée par les traitements technologiques qui leur sont appliqués ou par des conditions de conservation inappropriées. La surcuisson ou le surséchage sont là aussi des facteurs bien connus de dégradation de la digestibilité des protéines, de l'amidon et des sucres, comme l'ont montré par exemple Fernandez et Parsons (1996) et Aburto et al (1998) sur le tourteau de soja, Rymer et Givens (2005) sur le tourteau de colza, Bhuiyan et al (2010) sur le maïs grain.

d) Toxicité due à des contaminants chimiques ou biologiques des matières premières

La qualité chimique ou microbiologique des matières premières peut être altérée du fait d'une contamination au champ, au stockage ou lors d'un traitement industriel. Guitart et al (2010) ont proposé une revue des substances impliquées dans les épisodes d'intoxication chez les volailles et le bétail. Pour les volailles, les pesticides, herbicides, fongicides sont les principaux toxiques chimiques identifiés. Le fongicide Thiram semble responsable de dyschondroplasie tibiale chez le poulet ( $\mathrm{Li}$ et al 2007, Guitart et al 2010). Il n'existe pas de normes officielles de qualité microbiologique (bactéries, moisissures, levures) des aliments pour animaux à l'exception des salmonelles et des entérobactéries pour les poules et dindes reproductrices (règlement 2160/03/CE; arrêté du 23/04/2007 en France) mais on peut trouver des recommandations (Herry et al 1987). Pour les mycotoxines, des normes (directive 2002/32/CE) et des recommandations (2006/576/CE) existent en UE pour les aflatoxines et certaines fusariotoxines. Les relations entre mycotoxinesdose-effets-espèce-âge ne sont pas clairement établies dans bien des cas (Leeson et al 1995, Girgis et Smith 2010). Dans l'ensemble, le poulet semble peu sensible aux fusariotoxines (DON, zéaralénone, moniliformine) naturellement retrouvées dans les céréales et leurs coproduits (Girgis et Smith 2010). Toutefois, un lien a été reporté avec la dyschondroplasie (Whitehead 1998).

\section{2 / Quelles solutions alimen- taires mettre en œuvre pour améliorer le bien-être du poulet ?}

En considérant ces prérequis satisfaits, différentes solutions alimentaires et nutritionnelles sont envisagées pour limiter l'apparition de désordres liés à 
l'apparition de difficultés locomotrices, de troubles digestifs ou métaboliques, d'agressions respiratoires et de la mortalité. Ces solutions portent sur la composition de l'aliment, son aspect physique, son mode de distribution ou encore sur l'expérience précoce des volailles.

\section{1 / Adapter la composition de l'aliment}

\section{a) Gérer des antagonismes}

Les caractéristiques nutritionnelles pouvant modifier la santé ou le confort du poulet, sont présentées dans le tableau 1. Il s'agit des teneurs en protéines et acides aminés, en fibres, électrolytes, minéraux et vitamines, intervenant, soit sur l'état des fientes et donc sur la qualité de la litière, soit sur l'état du squelette.

La difficulté à adapter la composition de l'aliment provient de la nécessité à gérer différents antagonismes car des caractéristiques favorables à un aspect du bien-être animal peuvent l'être moins pour un autre. En effet, une déficience en protéines ou en acides aminés est perçue très rapidement par les poulets qui augmentent alors leur activité pour rechercher des nutriments complémentaires (Bizeray et al 2002, Bouvarel et al 2008). Des aliments pauvres en protéines ou en acides aminés mais aussi la restriction alimentaire peuvent ainsi favoriser les déplacements des animaux. Une déficience serait-elle alors un facteur de bien-être chez des poulets présentant naturellement une faible activité locomotrice? L'incidence des anomalies de la démarche est en effet en partie due à un manque d'activité physique (Bizeray et al 2004).

Un autre exemple porte sur les apports en calcium (Ca) et phosphore (P), constituants majeurs de la minéralisation osseuse. Le P est apporté en quantité de plus en plus faible pour des raisons économique et environnementale. Pourtant un apport conjoint et équilibré de ces deux éléments est nécessaire à la croissance de l'os, le Ca jouant un rôle déterminant sur la disponibilité du P (Narcy et al 2009).

b) Améliorer l'utilisation digestive de l'aliment et réduire les troubles digestifs

La fraction indigestible de l'aliment, qu'elle soit de nature protéique, lipidique ou glucidique est un facteur prédisposant aux désordres digestifs (transit accéléré, fermentation, enrichissement en eau, dysmicrobisme) susceptibles de dégrader la qualité de la litière et d'endommager l'intégrité physique des poulets (tableau 1).
Différents procédés sont connus pour améliorer la digestibilité des ingrédients de la ration :

- La formulation en acides aminés digestibles équilibrés («protéine idéale») qui permet une réduction du taux de protéines indifférenciées et limite ainsi l'apport d'azote non retenu. Ceci est facilité par l'ajout d'acides aminés obtenus par synthèse chimique ou fermentation (Leclercq 1996, Huyghebaert et al 2003):

- Le choix de la nature des ingrédients avec l'utilisation de variétés culturales plus digestibles, par exemple des pois moins riches en parois insolubles et en facteurs antitrypsiques (Gabriel et al 2007) ou des blés de faible dureté (Carré et al 2003, Skiba et al 2003);

- L'incorporation de fibres insolubles peu ou non fermentescibles (cosses d'avoine, coques de soja) permet un abaissement du $\mathrm{pH}$ stomacal, un ralentissement du transit digestif et une meilleure digestion de l'aliment (Gonzales-Alvarado et al 2008) (figure 1);

- Le traitement technologique des matières premières :

Les caractéristiques granulométriques des matières premières broyées sont connues pour influencer leur digestibilité (Carré 2000). Toutefois les recommandations qui peuvent en découler sont loin d'être univoques. Pour les céréales, la taille des particules est négativement corrélée à la digestibilité de l'amidon (Carré et al 2003) mais des particules grossières, voire des grains de blé ou de sorgho entiers, sont favorables au développement du gésier, au ralentissement du transit digestif et à la digestibilité de l'énergie (Gabriel et al 2008, Rodgers et al 2009). Le simple aplatissage des grains va aussi dans ce sens (Rodgers et al 2009). Une mouture fine est d'ailleurs favorable à l'apparition d'entérite nécrotique (Yegani et Korver 2008). L'utilisation de grains de blé entiers limiterait l'ingestion de litière par le poulet (Svihus et al 2010). Pour les dicotylédones données sous forme d'aliment farine, une mouture fine semble améliorer la digestibilité, bien que cela ne soit pas toujours observé (Valencia et al 2009) mais après granulation, l'effet propre de la mouture disparaît (Carré 2000).

Le traitement thermique ou hydrothermique qui élimine un certain nombre de FAN, notamment des oléoprotéagineux (Clarke et Wiseman 2007). Il est néanmoins important de le contrôler en testant par exemple la solubilité des protéines dans la potasse ou la teneur en facteurs antitrypsiques résiduels (Perilla et al 1997) car ces procédés réduisent parfois la digestibilité des protéines ou de l'amidon (Abd El-Khalek et Janssens 2010).

Le «trempage» des grains ou la distribution sous forme humide ou de soupe fermentée de l'aliment améliore sa digestibilité (Forbes et al 2005). Cela a été appliqué aussi au tourteau de colza (Chiang et al 2010).

\section{- Intérêt des additifs et autres ingré- dients}

L'utilisation d'enzymes sous forme d'additifs enregistrés (protéases, xylanases, glucanases, amylases...) ou sous forme de matières premières (céréales) fermentées riches en activités diverses non quantifiées est bien connue pour

Figure 1. Impact de l'introduction de fibres insolubles dans l'alimentation de poulets Cobb 500, de 1 à 21 jours d'âge à raison de 3\% d'enveloppes de graines de soja ou d'avoine, sur le pH des digesta du gésier, de l'EMAn et de la rétention azotée apparente (Gonzales-Alvarado et al 2008).

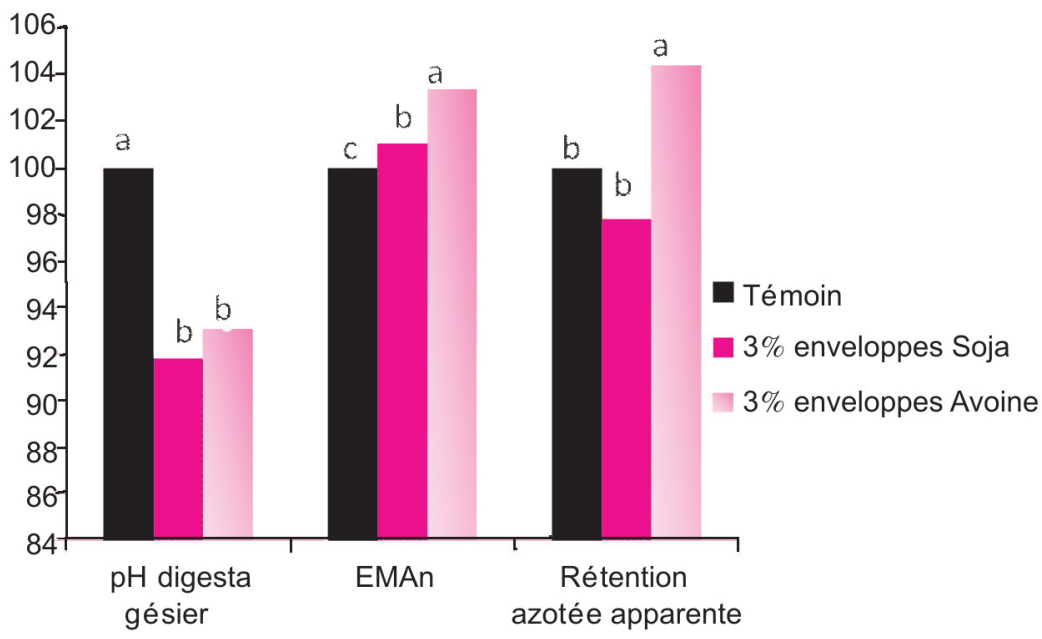

Les résultats sont exprimés en base 100 (Témoin) 
Tableau 1. Caractéristiques nutritionnelles des aliments pouvant influencer l'apparition de troubles digestifs et locomoteurs du poulet de chair. Actions correctrices possibles.

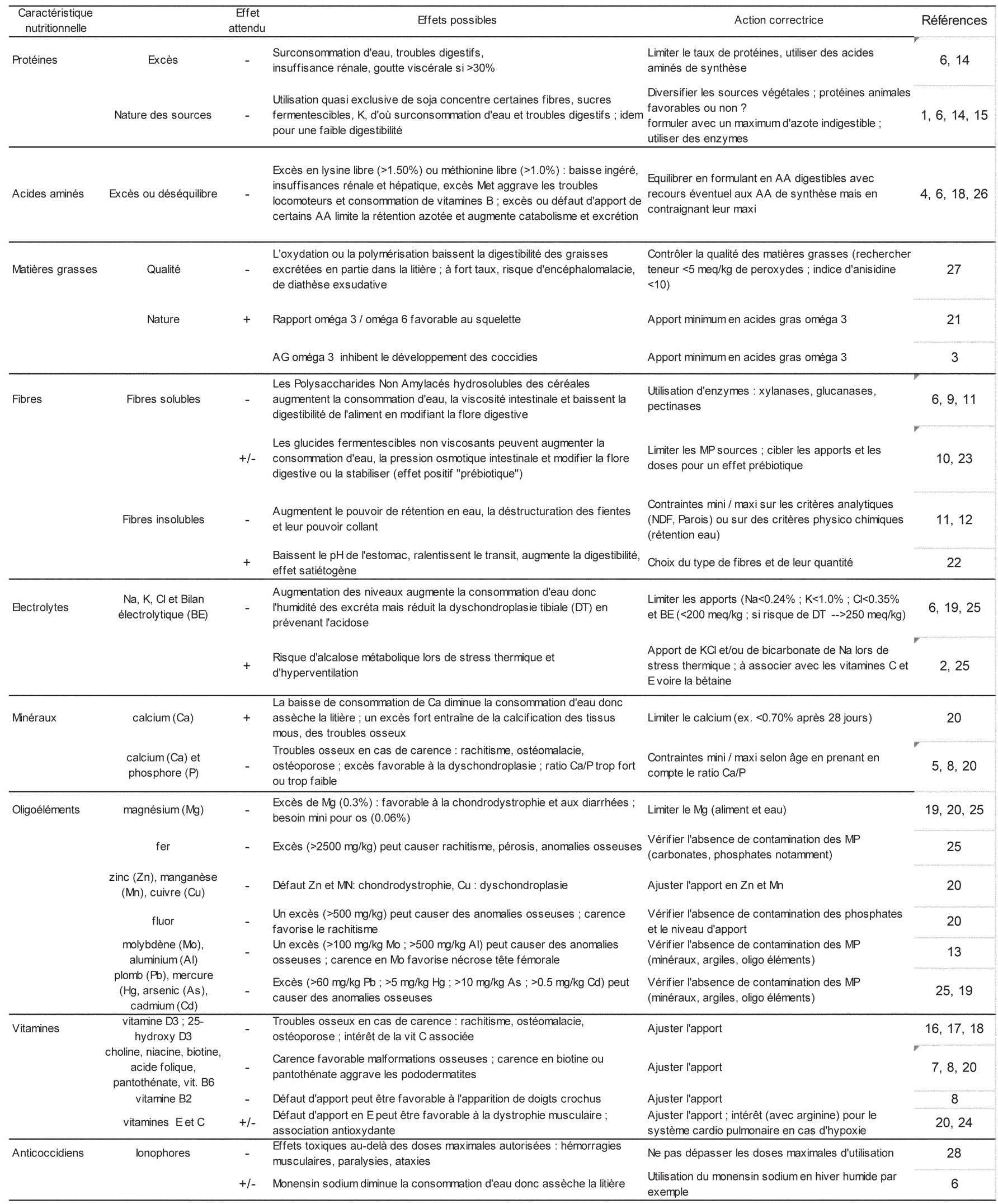

1 Manning et al 2007 ; 2 Sayed et Scott 2007 ; 3 Crevieu-Gabriel et Naciri 2001 ; 4 Dahiya et al 2007 ; 5 Narcy et al, 2009 ; 6 Francesch 2005 ; 7 Harms et al 1977 ; 8 Leterrier et al 1998; 9 Gao et al 2008; 10 Karr-Lilienthal et al 2005; 11 Carré et al 1995; 12 Carré et al $1994 ; 34$ Sauveur 1988; 14 Nagaraj et al 2007 ; 15 Eichner et al 2007; 16 Lohakare et al 2005 ; 17 Edwards 2000 ; 18 Whitehead 1998 ; 19 Oviedo-Rondon et al 2006 ; 20 Waldenstedt 2006 ; 21 Fleming 2008 ; 22 Jimenez-Moreno et al 2009 ; 23 Rehman et al 2009 ; 24 Bautista-Ortega et Ruiz-Feria 2010 ; 25 Leeson et al 1995 ; 26 Summers 1995 ; 27 Qureshi 2002 ; 28 Fowler 1995. 
améliorer la digestibilité globale de l'aliment (Parr 2011). De très nombreuses études et synthèses ont été publiées sur les effets d'ingrédients ou d'extraits très variés d'origine végétale, de leur copie synthétique, de microorganismes (bactéries, levures) sur les processus digestifs et fermentaires intestinaux ainsi que sur l'orientation des profils de la population microbienne digestive qui est un élément relativement stable et difficile à modifier (Gabriel et al 2003). Il est difficile d'appréhender réellement le succès de leur utilisation vis-à-vis du bien-être du poulet et en particulier du maintien d'une litière de qualité. C'est probablement pour cette raison que l'application pratique sur le terrain se traduit avant tout par des «recettes» qui doivent démontrer qu'elles donnent un avantage technico-économique réel. Ces «recettes» sont le plus souvent réalisées à partir des ingrédients suivants :

- les prébiotiques : sucres complexes de réserve (inuline), oligosaccharides produits par hydrolyse ou par synthèse, extraits de parois de levures (FOS, MOS ; Rehman et al 2009) avec parfois des conclusions négatives (Biggs et al 2007) ! L'inuline augmenterait la rétention minérale osseuse (Ortiz et al 2009) ;

- les probiotiques : préparations de un ou plusieurs microorganismes associés, destinées à prévenir le développement de bactéries pathogènes en «occupant» la place et à interagir favorablement avec le tube digestif (mucus, enzymes digestives, production d'acides gras volatils ; Gabriel et al 2005) ;

- les symbiotiques : qui associent prébiotique et probiotique (Yang et al 2009);

- les extraits végétaux : des extraits de châtaignier pourraient limiter la teneur en azote de la litière (Schiavone et al 2008); de multiples extraits (notamment des huiles essentielles) ou plantes entières ont été testées vis-à-vis de leur activité bactéricide ou bactériostatique in vitro avec des impacts parfois limités sur l'animal (Windish et al 2008, Brenes et Roura 2010, Tiihonen et al 2010) ;

- les enzymes : les xylanases notamment peuvent interagir avec le tractus digestif mais leurs effets sur la flore ou les fermentations intestinales ne sont pas clairs (Kocher 2003, Gao et al 2008). Nagaraj et al (2007) indiquent que l'utilisation d'un complexe enzymatique $(\alpha$-galactosidase, amylase, cellulase, protéase et pentosanase) permet de réduire la sévérité des pododermatites du poulet ;

- les acides organiques peuvent aussi avoir un rôle antimicrobien d'intérêt, en particulier l'acide butyrique, l'hydroxyanalogue de la méthionine et les acides protégés par micro-encapsulation pour court-circuter le tube digestif proximal, ainsi que les coccidiostatiques ionophores (Choct 2009, Dahiya et al 2007, Van Immerseel et al 2003) ;

- enfin, les acides gras oméga 3 ou l'artémisine inhibent le développement des coccidies, susceptibles de provoquer des lésions intestinales et des entérites (Crevieu-Gabriel et Naciri 2001).

\section{c) Prévenir les troubles métaboliques}

Parmi les nombreux troubles métaboliques (Leeson et al 1995, Julian 2005), certains peuvent être corrigés par l'alimentation. Dans le tableau 1 nous avons évoqué les déformations du squelette; citons aussi le stress thermique et l'utilisation bénéfique d'électrolytes $\left(\mathrm{NaHCO}_{3}, \mathrm{KCl}\right)$ (Bouvarel et al 1998) avec ou non les vitamines $\mathrm{C}$ et $\mathrm{E}$ contre le stress oxydatif associé. Un bon équilibre hydrominéral est aussi favorable au contrôle du syndrome «mort subite». Le ralentissement de la croissance par la distribution d'aliments en farine permet de limiter l'apparition d'ascites (Leeson 2007). De plus, concernant l'interaction nutrition et immunité, s'il est clair que l'alimentation peut supporter le système immunitaire dans son rôle de défense contre les agressions, beaucoup d'études sur des modèles sont nécessaires avant de pouvoir donner des normes nutritionnelles (Klasing 2007).

\section{2 / Adapter la présentation physique de l'aliment}

a) Limiter l'ingestion par l'aspect physique de l'aliment

La gestion de la taille des particules (farine ou miette plutôt que granulé) peut être un moyen de limiter la consommation et donc d'éviter une trop forte croissance des poulets susceptible d'engendrer de la mortalité. La présentation physique de l'aliment et notamment la taille des particules a un effet important sur la prise alimentaire. Les volailles consomment les particules suffisamment grosses pour être saisies efficacement par le bec (Rogers 1995), ces préférences correspondant à une optimisation énergétique (bénéfice/coût) du comportement alimentaire (Collier et Johnson 2004). Dans la pratique, les particules sont présentées le plus souvent sous forme de miettes dans le jeune âge puis de granulés. La granulation, par son action de compactage, permet d'améliorer l'efficacité de la prise alimentaire par le bec chez le poulet à croissance rapide (Nir et al 1994a et b, Quentin et al 2004, Svihus et al 2004, Linares et Huang 2010). Chagneau et al (2009) indiquent que la consommation est réduite de $22 \%$ avec de la farine comparée à du granulé. Dans cette même expérience, la teneur en matière sèche des fientes est augmentée significativement avec la farine. La présentation de l'aliment sous forme de farine a permis dans cette situation de réduire l'excrétion d'eau, probablement du fait d'un allongement du temps de transit total lié à une rétention plus longue dans le gésier. L'aliment granulé induit des effets mécaniques beaucoup plus limités qu'avec la farine probablement du fait de la diminution de la taille des particules lors du process mis en jeu pour la granulation (Svihus et al 2004).

b) Limiter la néophobie alimentaire pour éviter les comportements redirigés vers la litière et les congénères

Une nouvelle livraison d'aliment en élevage peut occasionner de la néophobie alimentaire avec une baisse ou un arrêt passager de consommation, associés à du picorage redirigé vers la litière (qui peut entraîner des diarrhées) ou les congénères (plaies, blessures) (Murphy 1977), avec parfois une plus forte consommation d'eau et donc une éventuelle dégradation de la litière. L'expression de ces comportements est en relation avec la modification des caractéristiques sensorielles des aliments. Des travaux récents réalisés notamment sur la dinde, montrent l'importance des propriétés visuelles et tactiles des particules alimentaires, en l'occurrence leur aspect (forme, couleur) (Lecuelle et al 2010) et leur rigidité (Laviron et al 2010).

Deux solutions peuvent être envisagées pour réduire la néophobie des volailles : réduire les contrastes visuels et tactiles lors d'un changement d'aliment, de manière à apporter aux volailles une «continuité sensorielle» ou encore augmenter la diversité sensorielle des aliments dès le plus jeune âge, les volailles étant capables d'apprentissage permettant ainsi une meilleure adaptation à un changement (Lecuelle 2011). La question est maintenant de savoir comment apporter au mieux cette «diversité».

\section{3 / Jouer sur les modalités de distribution de l'aliment}

La distribution d'aliments complets ad libitum est le cas le plus fréquemment observé en France pour l'élevage du poulet de chair. D'autres techniques de distribution peuvent être pratiquées en modulant la quantité et/ou le temps d'accès à l'aliment ou encore en laissant un choix plus ou moins dirigé au poulet dans l'espace et le temps.

a) Restriction alimentaire au démarrage

Les facteurs alimentaires stimulant la vitesse de croissance, surtout en phase 
de démarrage favorisent l'apparition de troubles locomoteurs. A ce titre, l'augmentation des apports énergétiques et protéiques est la plus néfaste (Leterrier et al 1998). La restriction alimentaire quantitative peut être utilisée en période de démarrage pour prévenir de déformations du squelette (Lee et Leeson 2001) mais aussi d'autres troubles comme les ascites (Tottori et al 1997, Arce-Menocal et al 2009) ou le syndrome de la mort subite (Blair et al 1993).

Néanmoins, la restriction quantitative au démarrage entraîne le plus souvent une diminution du gain de poids à l'abattage. De plus, étant donné le comportement hyperphagique du poulet de chair à croissance rapide, elle peut apparaître comme non favorable au bien-être animal.

La restriction alimentaire qualitative, qui consiste à diluer les principaux apports nutritionnels, peut être également pratiquée au démarrage. Avec une réduction uniquement de la teneur énergétique de l'aliment au démarrage, Lippens et al (2002) indiquent une tendance à la réduction de la mortalité due au syndrome de mort subite, sans effet sur le poids à l'abattage.

\section{b) Mise à jeun en cas de forte chaleur}

En cas de pics de chaleur avec des poulets en fin d'élevage, il est conseillé de retirer l'aliment un peu avant et pendant les heures les plus chaudes de la journée, de manière à ce que les animaux réduisent leur production de chaleur, ce qui conduit à une baisse de la température corporelle et de la mortalité (Francis et al 1991, Vilarino et al 1996, Yalcin et al 2001). Cette technique peut être complétée en période estivale par la distribution de sels dans l'eau de boisson (cf. 2.1.c).

\section{c) Alimentation séquentielle}

La gestion d'un régime alimentaire complexe est un outil qui reste encore assez peu exploré à ce jour. D'autres techniques de distribution de l'aliment permettent non pas de distribuer «un» aliment mais «des» aliments, recréant ainsi partiellement des conditions d'alimentation plus variées. La technique de la distribution séquentielle consiste à distribuer de manière cyclique deux aliments différents, dont la combinaison permet des apports nutritionnels équilibrés. L'alimentation séquentielle donne la possibilité au poulet d'exprimer des tendances naturelles de consommation, contraintes toutefois par la durée de dis- tribution des aliments. Le poulet est en effet poussé à consommer un aliment moins préféré si la durée d'accès est longue. L'avantage est de moduler les apports nutritionnels tout au long de la vie des volailles avec seulement deux aliments.

L'utilisation de deux aliments de caractéristiques énergétique et protéique différentes se révèle efficace en conditions de production, avec l'absence de dégradation du poids et de l'efficacité alimentaire (Bouvarel et al 2004, 2008 ) tout en améliorant le bien-être des poulets en réduisant les troubles locomoteurs, du fait certainement d'une activité plus importante (Bizeray et al 2002, Bouvarel et al 2004, Leterrier et al 2005). Cette technique peut être utilisée aussi avec des céréales entières, ce qui permet de réduire les coûts de fabrication et de transport (Noirot et al 1999). La technique du mélange, pratiquée en Europe du nord, est intéressante en termes d'économie et de bien-être, mais peut induire un tri particulaire augmentant l'hétérogénéité du poids des animaux.

\section{4 / Peut-on pré-conditionner les poulets?}

En enrichissant l'alimentation de la poule en caroténoïdes par exemple (canthaxanthine, lutéine), il semble possible d'augmenter la viabilité du poussin en augmentant sa résistance aux stress oxydatifs (Rocha et al 2010). De même, la teneur en vitamine D3 de l'aliment de la poule peut modifier l'apparition et la gravité de la dyschondroplasie tibiale chez le poulet (Driver et al 2006). L'enrichissement du vitellus en acides gras oméga 3 grâce à l'aliment maternel peut augmenter la résistance osseuse (Dibner et al 2007).

Si les études portant sur la nutrition in ovo par injection dans l'amnios visent souvent à augmenter les capacités digestives et le développement musculaire du poulet (Gous 2010), cette approche pourrait également favoriser le développement et la résistance osseuse (Ferket 2010).

Par ailleurs, les poulets semblent capables de mémoriser des expériences olfactives durant la vie embryonnaire et consommer préférentiellement un aliment dont ils ont expérimenté l'odeur (Bertin et al 2010). Ceci est intéressant dans la mesure où une alimentation néonatale et un abreuvement précoces favo- risent la survie et le développement du poussin (Willemsen et al 2010). Par ailleurs un enrichissement en lysine de cet aliment pourrait favoriser la tolérance des poulets à des aliments moins concentrés en protéines en phase finition d'où une meilleure litière. Une carence en phosphore dans les premiers jours de vie favoriserait l'utilisation du phosphore phytique; cette «empreinte nutritionnelle» est probablement à relier à la notion d'adaptation ou de programmation épigénétique, traduisant l'influence (héritable) de l'environnement (aliment, conditions d'incubation...) sur l'activité des gènes sans modifier le génome (Jammes et Renard 2010).

\section{Conclusion}

La conduite alimentaire permet de limiter l'apparition de troubles locomoteurs, digestifs et métaboliques qui sont autant de facteurs d'altération de ce que l'on peut définir, au-delà de considérations émotionnelles, comme le bien-être des poulets de chair. Elle peut permettre par ailleurs d'enrichir le milieu de vie des animaux. Certains choix nutritionnels peuvent toutefois se révéler paradoxalement améliorateurs ou aggravants selon le critère de bien-être retenu. De plus, au regard du bien-être animal, la conduite alimentaire ne représenterait qu'un levier avec la gestion de l'ambiance autour de l'animal et le choix du génotype. La sélection génétique conduit aujourd'hui à produire des poulets à très fort potentiel de croissance avec des effets négatifs induits sur l'expression des comportements et les capacités d'adaptation.

Pour le moment, l'alimentation s'adapte à cette évolution génétique essentiellement par ajustement de la composition de l'aliment et par la gestion de la présentation physique. Des marges de progrès sont encore possibles en jouant sur les modalités de distribution. Les effets de la sélection génétique sur le comportement alimentaire et l'activité des poulets méritent d'être pris en compte dans les recherches à venir.

Enfin, globalement, une réflexion est à mener en termes de sélection pour faire évoluer le matériel génétique, car la préservation du bien-être doit certainement passer par une amélioration des capacités d'adaptation des poulets. Le pré-conditionnement apparaît aussi dans ce cadre comme une voie prometteuse. 


\section{Références}

Abd El-Khalek E., Janssens G.P.J., 2010. Effect of extrusion processing on starch gelatinisation and performance in poultry. World's Poult. Sci. J., 66, 53-63.

Aburto A., Vazquez M., Dale N.M., 1998. Strategies for utilizing overprocessed soybean meal: 1. amino acid supplementation, choline content, and metabolizable energy. J. Appl. Poult. Res., 7, 189-195.

Allain V., Mirabito L., Arnould C., Colas M., Le Bouquin S., Lupo C., Michel V., 2009. Skin lesions in broiler chickens measured at the slaughterhouse: relationships between lesions and between their prevalence and rearing factors. Brit. Poult. Sci., 50, 407-417.

Arce-Menocal J., Avila-Gonzalez E., LopezCoello C., Garibay-Torres L., Martinez-Lemus L.A., 2009. Body weight, feed-particle size, and ascites incidence revisited. J. Appl. Poult. Res., 18, 465-471.

Arnould C., 2005. Bien-être du poulet de chair : mesures, problèmes rencontrés et moyens d'action. 6emes Journées Rech. Avic., St-Malo, France, $7 \mathrm{p}$

Baker D.H., 2008. The thrill of discovery: by design and by accident. In: Proceedings Arkansas Ann. Nutri. Conf., 9-11 sept., Rogers, Arkansas, USA, 8p.

Bautista-Ortega J., Ruiz-Feria C.A., 2010. L-Arginine and antioxydant vitamins $\mathrm{E}$ and $\mathrm{C}$ improve the cardiovascular performance of broiler chickens grown under chronic hypobaric hypoxia. Poult. Sci., 89, 2141-2146.

Bertin A., Calandreau L., Arnould C., Nowak R., Levy F., Noirot V., Bouvarel I., Leterrier C., 2010. In Ovo olfactory experience infuences post-hatch feeding behaviour in young chickens. Ethology, 116, 1-11.

Bhuiyan M.M., Iji P.A., Islam A.F., Mikkelsen L.L., 2010. Variation in nutrient composition and structure of high-moisture maize dried at different temperatures. Aust. Poult. Sci. Symp., 136-139.

Biggs P., Parsons C.M., Fahey G.C., 2007. The effects of several oligosaccharides on growth performance, nutrient digestibilities, and cecal microbial populations in young chicks. Poult. Sci., 86, 2327-2336.

Bizeray D., Leterrier C., Constantin P., Picard M., Faure J.M., 2002. Sequential feeding can increase activity and improve gait score in meat-type chickens. Poult. Sci., 81, 1798-1806.

Bizeray D., Faure J.M., Leterrier C., 2004. Faire marcher le poulet : pourquoi et comment. INRA Prod. Anim., 17, 45-57.

Blair R., Newberry R.C., Gardinen E.E., 1993. Effects of lighting pattern and dietary tryptophan supplementation on growth and mortality in broilers. Poult. Sci., 72, 495-502.

Bouvarel I., Fargeas E., Ferchal E., Roffidal L., Guillaumin J. M., Saint Jan B. de, Tesseraud S., 1998. In : La prévention du coup de chaleur en aviculture, S.T.A., 24, 15-18.

Bouvarel I., Barrier-Guillot B., Larroude P., Boutten B., Leterrier C., Merlet F., Vilarino M. Roffidal L. Tesseraud S. Castaing J., Picard M., 2004. Sequential feeding programs for broiler chickens: twentyfour- and forty-eight-hour cycles. Poult. Sci. $83,49-60$.
Bouvarel I., Chagneau A.M., Lescoat P., Tesseraud S., Leterrier C., 2008. Forty-eighthour cycle sequential feeding with diets varying in protein and energy contents: adaptation in broilers at different ages. Poult. Sci., 87, 196-203.

Brenes A., Roura E., 2010. Essential oils in poultry nutrition: main effects and modes of action. Anim. Feed Technol., 158, 1-14.

Carré B., 2000. Effets de la taille des particules alimentaires sur les processus digestifs chez les oiseaux d'élevage. INRA Prod. Anim., 13, 131-136.

Carré B., Gomez J., Melcion J.P., Giboulot B., 1994. La viscosité des aliments destinés à l'aviculture. Utilisation pour prédire la consommation et l'excrétion d'eau. INRA Prod. Anim., 7, 369-379.

Carré B., De Monredon F., Melcion J.P., Gomez J., 1995. Qualité de la litière en aviculture. Aliments et caractéristiques physiques des excreta. INRA Prod. Anim., 8, 331-334.

Carré B., Muley N., Guillou D., Signoret C. Oury F.X., Gomez J., 2003. Effet de la dureté des blés sur la digestion de leur amidon chez les poulets de 3 semaines. 5èmes Journ. Rech. Avicole., Tours, France, 4p.

Cassy S.M.P, Crochet S., Derouet M., Keisler D.H., Taouis M., 2004. Peripheral leptin effect on food intake in young chickens is influenced by age and strain. Dom. Anim. Endocrinol., 27, 51-61.

Chagneau A.M., Lecuelle S., Lescoat P., Guillaumin J.M., Quentin M., Bouvarel I., 2009. Effets du mode de distribution et de la présentation de l'aliment sur les performances du poulet de chair à croissance rapide 8 èmes Journ. Rech. Avicole., St-Malo, France, 4p.

Chiang G., Lu W.Q., Piao X.S., Hu J.K., Gong L.M., Thacker P.A., 2010. Effects of feeding solid-state fermented rapeseed meal on performance, nutrient digestibility, intestinal ecology and intestinal morphology of broiler chickens. Asian-Aust. J. Anim. Sci., 23, 263-271.

Choct M., 2009. Managing gut health through nutrition. Brit. Poult. Sci., 50, 9-15.

Clarke E., Wiseman J., 2007. Effects of extrusion conditions on trypsin inhibitor activity of full fat soybeans and subsequent effects on their nutritional value for young broilers. Brit. Poult. Sci., 48, 703-712.

de Coca Sinova A., Jimenez-Moreno E., Gonzalez-Alvarado J.M., Frikha M., Lazaro R., Mateos G.G., 2010. Influence of source of soybean meal and lysine content of the diet on performance and total tract apparent retention of nutrients in broilers from 1 to 36 days of age. Poult. Sci., 89, 1440-1450.

Collier G., Johnson D.F., 2004. The paradox of satiation. Physiol. Behav., 82, 149-153.

Crevieu-Gabriel I., Naciri M., 2001. Effet de l'alimentation sur les coccidioses chez le poulet. INRA Prod. Anim., 14, 231-246.

D'Mello J.P.F., 2000. Anti-nutritional factors and mycotoxins In: Farm Animal Metabolism and Nutrition. J.P.F. D’Mello (Ed). 383-405.

Dahiya J.P., Hoehler D., Van Kessel A.G., Drew M.D., 2007. Effect of different dietary methionine sources on intestinal microbial populations in broiler chickens. Poult. Sci., 86, 2358-2366.

Dibner J.J., Richards J.D., Kitchell M.L. Quiroz M.A., 2007. Metabolic challenges and early bone development. J. Appl. Poult. Res., $16,126-137$

Directive 2002/32/CE du parlement européen et du Conseil du 7 mai 2002 sur les substances indésirables dans les aliments pour animaux. Journal officiel, 30 mai 2002, L140, $10-21$.

Directive 2007/43/CE du Conseil du 28 juin 2007 fixant des règles minimales relatives à la protection des poulets destinés à la production de viande. Journal officiel, 12 juillet 2007 , L 182, 19-28.

Dozier W.A., 2003. How much water is enough? Poult. Dig. Online, 3, 35.

Drew M.D., Syed N.A., Goldade B.G. Laarveld B., Van Kessel A.G., 2004. Effects of dietary protein source and level on intestinal populations of Clostridium perfringens in broiler chickens. Poult. Sci., 83, 414-420.

Driver J.P., Atencio A., Pesti G.M., Edwards H.M. Jr., Bakalli R.I., 2006. The effect of maternal dietary vitamin D3 supplementation on performance and tibial dyschondroplasia of broiler chicks. Poult. Sci., 85, 39-47.

Eichner G., Vieira S.L., Torres C.A. Coneglian J.L.B., Freitas D.M., Oyarzabal O.A., 2007. Litter moisture and foodpad dermatitis as affected by diets formulated on an all-vegetable basis or having the inclusion of poultry by-product. J. Appl. Poult. Res., 16, 344-350

Edwards H.M. Jr, 2000. Nutrition and skeletal problems in poultry. Poult. Sci., 79, 10181023.

Esmail S.H.M., 1998. Raw soybeans have limited nutritional value. World Poult., 14, 20-22.

Ferket P.R., 2010. Poultry nutrition moves towards higher standards. In: World poultry.net 18/06/10.

Fernandez S.R., Parsons C.M., 1996. Bioavailability of digestible lysine in heatdamaged soybean meal for chick growth. Poult. Sci., 75, 224-231.

Fleming R.H., 2008. Nutritional factors affecting poultry bone health. Proc. Nutr. Soc., 67, 177-183.

Forbes M., Slade R.D., Yalda A.Y., 2005. Wet feeding of young chicks. Proceedings Aust. Poult. Sci. Symp., 4p.

Fowler N.G., 1995. Anticoccidial compendium, safety, toxicity, side effects, incompatibilities. In: Anticoccidial compendium. Fowler N.G. (ed), 73p.

Francesch M., 2005. Facteurs nutritionnels modifiant l'humidité et la qualité des excreta et de la litière en volailles. 6èmes Journ. Rech. Avicole., St-Malo, France, 146-153.

Francis C.A., Macleod M.G., Anderson J.E.M., 1991. Alleviation of acute heat stress by food withdrawal or darkness. Brit. Poult. Sci. 32, 219-225.

Gabriel I., Mallet S., Lessire M., 2003. La microflore digestive : une composante oubliée de la nutrition des volailles. $5^{\text {èmes }}$ Journ. Rech. Avicole, Tours, France, 8p. 
Gabriel I., Mallet S., Sibille P., 2005. La microflore digestive des volailles : facteurs de variation et conséquences pour l'animal. INRA Prod. Anim., 18, 309-322.

Gabriel I., Lessire M., Juin H., Burstin J., Duc G., Quillien L., Thibault J.N., Ganier P., Mézière N., Leconte M., Hallouis J.M., Cassecuelle F., Marget P., Sève B., 2007. Origine de la variation de la digestion des protéines de pois (Pisumsativum L.) chez le poulet. 7 èmes Journ. Rech. Avicole, Tours, France, 5p.

Gabriel I., Mallet S., Leconte M. Travel A., Lalles J.P., 2008. Effects of whole wheat feeding on the development of the digestive tract of broiler chickens. Anim. Feed Sci. Technol. $142,144-162$

Gao F., Jiang Y., Zhou G.H., Han Z.K., 2008. The effects of xylanase supplementation on performance, characteristics of the gastrointestinal tract, blood parameters and gut microflora in broilers fed wheat-based diets. Anim. Feed Sci. Technol., 142, 173-184.

Girgis G.N., Smith T.K., 2010. Comparative aspects of Fusarium mycotoxicoses in poultry fed diets containing naturally contaminated grains. World's Poult. Sci. J., 66, 65-86.

Gonzales-Alvarado J.M., Jimenez-Moreno E., Valencia D.G., Lazaro R., Mateos G.G., 2008. Effects of fiber source and heat processing of the cereal on the development and $\mathrm{pH}$ of the gastrointestinal tract of broilers fed diets based on corn or rice. Poult. Sci., 87, 17791795.

Gous R.M., 2010. Nutritional limitations on growth and development in poultry. Liv. Sci., $130,25-32$.

Guitart R, Croubels S, Caloni F, Sachana M., Davanzo F., Vandenbroucke V., Berny P., 2010. Animal poisoning in Europe: part 1: farm livestock and poultry. Vet. J., 183, 249-254.

Halley J.T., 2007. Recommendations for the modern broiler. Cobb Vantress Inc, dossier technique, $12 p$.

Harms R.H., Damron B.L., Simpson C.F., 1977. Effect of wet litter and supplemental biotin and/or whey on the production of food pad dermatitis in broilers. Poult. Sci., 56, 291-296.

Herry M.P., Michard J., Beaudoin B., Lelay Y., 1987. L'analyse microbiologique de matières premières pour l'alimentation animale et son interprétation. Possibilités d'établissement de normes. Microbiol. Alim. Nutr., 5, 21-43.

Huyghebaert G., Coenen H., Le Bellego L., 2003. Impact de la teneur en protéines et du profil en acides aminés de l'aliment sur les performances zootechniques du poulet de chair. 5 èmes Journ. Rech. Avicole, Tours. France, $4 \mathrm{p}$.

ITAVI, 2007. Eau de boisson en élevage avicole, un levier majeur de réussite. Document Technique 12p

Jammes H., Renard J.P., 2010. Epigénétique et construction du phénotype, un enjeu pour les productions animales ? In : Robustesse, rusticité, flexibilité, plasticité, résilience... les nouveaux critères de qualité des animaux et des systèmes d'élevage. Sauvant $\mathrm{D}$, Perez J.M. (Eds). Dossier, INRA Prod. Anim., 23, 23-42.

Jimenez-Moreno E., Gonzales-Alvarado J.M., Lazaro R., Mateos G.G., 2009. Effects of type of cereal, heat processing of the cereal, and fiber inclusion in the diet on gizzard $\mathrm{pH}$ and nutrient utilization in broilers at different ages. Poult. Sci., 88, 1925-1933.
Julian R.J., 2005. Production and growth related disorders and other metabolic diseases of poultry - a review. Vet. J., 169, 350-369.

Karr-Lilienthal L.K., Kadzere C.T. Grieshop C.M., Fahey G.C. Jr., 2005. Chemical and nutritional properties of soybean carbohydrates as related to nonruminants - a review. Liv. Prod. Sci., 97, 1-12.

Klasing K.C., 2007. Nutrition and the immune system. Brit. Poult. Sci., 48, 525-537.

Kocher A., 2003. Nutritional manipulation of necrotic enteritis outbreak in broilers. Rec. Adv. Anim. Nutr. Austral., 14, 111-116.

Laviron F., Chagneau A.M., Lecuelle S. Lescoat P., Leterrier C., Bouvarel I., 2010. Pellet hardness effects short-term intake during diet change-over in turkeys. XIII ${ }^{\text {th }}$ Eur. Poult Conf., Aug. 24-26, Tours, France, 457.

Leclercq B., 1996. Les rejets azotés issus de ',aviculture : importance et progrès envisageables. INRA Prod. Anim., 9, 91-101.

Lecuelle S., 2011. Comportement alimentaire des dindes en lien avec les caractéristiques visuelles et tactiles des aliments. Thèse Université François Rabelais, Tours, France, $218 \mathrm{p}$.

Lecuelle S., Bouvarel I., Chagneau A.M Laviron F, Lescoat P. Leterrier C. 2010. Colour effects on diet change-over in turkeys XIIIth Eur. Poult. Conf., Aug. 24-26, Tours, France, 813 .

Lee K.L., Leeson S., 2001. Performance of broilers fed limited quantities of feed or nutrients during seven to fourteen days of age. Poult. Sci., 80, 446-454.

Leeson S., 2007. Metabolic challenges: past, present, and future. J. Appl. Poult. Res., 16, 121-125.

Leeson S., Summers J.D., 2000. Feeding systems for poultry. In: Feeding systems and feed evaluation models. Theodorou M.K. France J. (Eds), reading, UK, 211-237.

Leeson S., Diaz G.J., Summers J., 1995. Poultry metabolic disorders and mycotoxins. University Books (Ed), 352p.

Leterrier C., Constantin P., Le Bihan-Duva E., Marché G., Nys Y., 1998. Troubles locomoteurs et qualité osseuse chez les volailles de chair. INRA Prod. Anim., 11, 125-130.

Leterrier C., Favreau F., Constantin P. Picard M., 2005. Effects of various lysine levels on growth and leg problem in meat-type chickens during sequential feeding. $7^{\text {th }}$ Eur. Symp. Poultry Welfare, Lublin, Pologne, Animal Science Papers and Reports, 241-248.

Li J., Bi D., Pan S., Zhang Y., 2007. Effect of diet with thiram on liver antioxidant capacity and tibial dyschondroplasia in broilers. Brit. Poult. Sci., 48, 724-728.

Linares L., Huang K., 2010. Effects of energy levels and different feed processing methods on performance of broilers. XIII ${ }^{\text {th }}$ Eur. Poult. Conf., Aug. 24-26, Tours, France, 5p.

Lippens M., Huyghebaert G., Van Tuyl O. De Groote1 G., 2002. Early and temporary qualitative, autonomous feed restriction of broiler chickens. Effects on performance characteristics, mortality, carcass and meat quality. Arch. Geflugelk., 67, 49-56.

Lohakare J.D., Kim J.K., Ryu M.H., Hahn T.W., Chae B.J., 2005. Effects of vitamin C and vitamin $\mathrm{D}$ interaction on the performance, immunity, and bone characteristics of commercial broilers. J. Appl. Poult. Res., 14, 670-678.

Lordelo M.M., Davis A.J., Calhoun M.C., Dale N.M., 2004. Performance of broilers receiving positive and negative isomers of gossypol and respective observations on tissue accumulation. WPSA Congress, Istanbul.

Manning L., Chadd S.A., Baines R.N., 2007. Key health and welfare indicators for broiler production. World's Poult. Sci. J., 63, 46-62.

Métayer J.P., Barrier-Guillot B., Skiba F., Crépon K., Bouvarel I., Marget P., Duc G., Lessire M., 2003. Valeur alimentaire et utilisation de différents types de féveroles chez le poulet et le coq adulte. 5èmes Journ. Rech. Avicole., Tours, France, 4p.

Montiel A., 2007. Qualité de l'eau en élevage avicole. 7 emmes Journ. Rech. Avicole, Tours, France, 455-459.

Murphy, L. 1977. Response of domestic fowl to novel food and objects. Appl. Anim. Ethol., 3, 335-349.

Nagaraj M., Wilson C.A.P., Hess J.B., Bilgili S.F., 2007. Effect of high-protein and all-vegetable diets on the incidence and severity of pododermatitis in broiler chickens. J. Appl. Poult. Res., 16, 52-61.

Narcy A., Létourneau-Montminy M.P., Magnin M., Nys Y., Jondreville C., 2009. Voies nutritionnelles d'économie de phosphore chez le poulet. 8 èmes Journ. Rech. Avicole, St-Malo, France, $8 \mathrm{p}$

Nielsen B.L., 2004. Behaviour aspects of feeding constraints: do broilers follow their out feelings ? Appl. Anim. Behav. Sci., 86, 251-260.

Nir I., Shefet G., Aaroni Y., 1994a. Effect of particle size on performance. 1. Corn. Poult. Sci., 73, 45-49.

Nir I., Twina Y., Grossman E., Nitsan Z., 1994b. Quantitative effects of pelleting on performance, gastrointestinal tract and behaviour of meat-type chickens. Brit. Poult. Sci., 35, 589-602.

Noirot V., Bouvarel I., Azam P., Roffidal L., Barrier-Guillot B., Castaing J., Picard M., 1999. Du blé entier en alimentation séquentielle en poulet «standard» : résultats zootechniques obtenus en élevage. S.T.A., 27, 2-11.

Nyamambi B., Ndlovu L.R., Naik Y.S., Kock N.D., 2007. Intestinal growth and function of broiler chicks fed sorghum based diets differing in condensed tannin levels. South Afric. J. Anim. Sci., 37, 202-214

Nys Y., 2001. Oligo-éléments, croissance et santé du poulet de chair. INRA Prod. Anim., $14,171-180$

Ortiz L.T., Rodriguez M.L. Alzueta C., Rebolé A., Trevino J., 2009. Effect of insulin on growth performance, intestinal tract sizes, mineral retention and tibial bone mineralisation in broiler chickens. Brit. Poult. Sci., 50, 325-332

Oviedo-Rondon E.O., Ferket P.R., Havenstein G.B., 2006. Nutritional factors that affect leg problems in broilers and turkeys. Avian and Poult. Biol. Rev., 17, 89-103, 115.

Parr T., 2011. Exogenous enzymes. Role and function in poultry diets. Proceedings Midwest Poultry Federation Convention. 16-17 mars, St Paul, Minnesota, USA, 8p.

Perilla N.S., Cruz M.P., De Belalcazar F. Diaz G.J., 1997. Effect of temperature of wet 
extrusion on the nutritional value of full-fat soyabeans for broiler chickens. Brit. Poult. Sci., 38, 412-416.

Qiao H.Y., Dahiya J.P., Classen H.L., 2008. Nutritional and physiological effects of dietary sinapic acid (4-hydroxy-3,5-dimethoxy-cinnamic acid) in broiler chickens and its metabolism in the digestive tract. Poult. Sci., 87, 719726.

Quentin M., Bouvarel I., Berri C., Le BihanDuval E., Baéza E., Jégo Y., Picard M., 2003. Growth, carcass composition and meat quality response to dietary concentrations in fast-, medium- and slow-growing commercial broilers. Anim. Res. 52, 65-77.

Quentin M., Bouvarel I., Picard M., 2004. Short and long term effects of feed form on fast- and slow-growing broilers. J. Appl. Poult. Res., 13, 540-548.

Qureshi A.A., 2002. Oxidative rancidity in poultry feed. Poult. Int., Mars, 38-42.

Rebolé A., Rodriguez M.L., Ortiz L.T., Alzueta C., Centeno C., Trevino J., 2002. Mucilage in linseed: effects on the intestinal viscosity and nutrient digestion in broiler chicks. J. Sci. Food Agric., 82, 1171-1176.

Recommandation 2006/576/CE de la Commission européenne du 17 août 2006 concernant la présence de déoxynivalénol, de zéaralénone, d'ochratoxine $\mathrm{A}$, des toxines $\mathrm{T}-2$ et HT-2 et de fumonisines dans les produits destinés à l'alimentation animale. Journal officiel, 23 Août 2006, L 229, 7-9.

Règlement 2160/2003/CE du Parlement européen et du Conseil du 17 novembre 2003 sur le contrôle des salmonelles et d'autres agents zoonotiques spécifiques présents dans la chaîne alimentaire. Journal officiel, 12 décembre 2003, L 325, 1-15.

Rehman H., Vahjen W., Kohl-Parisini A., Ijaz A., Zentek J., 2009. Influence of fermentable carbohydrates on the intestinal bacteria and enteropathogens in broilers. World's Poult. Sci. J., 65, 75-89.

Rocha J.S.R., Lara L.J.C., Baiao N.C., Vasconcelos R.J.C., Barbosa V.M., Pompeu M.A., Fernandes M.N.S., 2010. Antioxydant properties of vitamins in nutrition of broiler breeders and laying hens. World's Poult. Sci. J., 66, 261-270.

Rodgers N., Iji P.A., Mikkelsen L.L., Svihus B., Hetland H., Choct M., 2009. Effect of grain particle size and milling method on broiler performance and apparent metabolisable energy. Aust. Poult. Sci. Symp., 133-136.
Rogers P.J., 1995. The development of the brain and behaviour in the chicken. CAB Int., Wallingford (Ed). UK, 95-110.

Rymer C., Givens D.I., 2005. Factors affecting the protein quality of rapeseed meal. Feed Compounder, 28-33.

Sayed M.A.M., Scott T.A., 2007 Maintaining electrolytes and water balance to alleviate heat stress in broiler chickens. Proc. Aust. Poult. Sci. Symp., 23-26.

Sauveur B., 1988. Lésions osseuses et articulaires des pattes des volailles : rôles de l'alimentation. INRA Prod. Anim., 1, 35-45.

Schiavone A., Guo K., Tassone S., Gasco L., Hernandez E., Denti R., Zoccarato I., 2008. Effects of a natural extract of chestnut wood on digestibility, performance traits, and nitrogen balance of broiler chicks. Poult. Sci., 87, 521527.

Shariatmadari F., Forbes J.M., 1993. Growth and food intake responses to diets of different protein contents and a choice between diets containing two concentrations of protein in broiler and layer strains of chicken. Brit. Poult. Sci., 34, 959-970.

Skiba F., Barrier-Guillot B., Métayer J.P. 2003. Effet de la teneur en protéines, du poids spécifique et de la teneur en amidon, de la dureté et de la germination sur la valeur alimentaire du blé chez le jeune poulet. 5èmes Journ. Rech. Avicole, Tours, France, 4p.

Summers J.D., 1995. Excess sulphur can have toxic effect on poultry. Feedstuffs, 16-35.

Svihus B., Sacranie A., Denstadli V., Choct M., 2010. Nutrient utilization and functionality of the anterior digestive tract caused by intermittent feeding and inclusion of whole wheat in diets for broiler chickens. Poult. Sci.,. 89, 2617-2625.

Svihus B., Kløvstada K.H., Pereza V., Zimonjaa O., Sahlströmb S., Schüllerc R.B., Jeksrudd W.K., Prestløkkene E., 2004. Physical and nutritional effects of pelleting of broiler chicken diets made from wheat ground to different coarsenesses by the use of roller mill and hammer mill. Anim. Feed Sci. Tech., 117, 281-293.

Tiihonen K., Kettuen H., Bento M.H.L., Saarinen M., Lahtinen S., Ouwehand A.C., Schulze H., Rautonen N., 2010. The effect of feeding essential oils on broiler performance and gut microbiota. Brit. Poult. Sci., 51, 381392.

Travel A., Chevalier D., Merlet F., Fulbert L., 2007. Facteurs de variation de la qualité bactériologique de l'eau en élevage de dindes. 7 èmes Journ. Rech. Avicole, Tours, France, 536540 .

Tottori J.R., Yamaguchi Y., Murakama M., Sato K., Uchida K., Tateyama S., 1997. The use of feed restriction for mortality control of chickens in broiler farms. Avian Dis., 41, 433437.

Valencia D.G., Serrano M.P., Lazaro R., Jimenez-Moreno E., Mateos G.G., 2009. Influence of micronization (fine grinding) of soya bean meal and full-fat soya bean on the ileal digestibility of amino acids for broilers. Anim. Feed Sci. Technol., 150, 238-248.

Van Immerseel F., De Buck J., Pasmans F., Haesebrouck F., Ducatelle R., 2003. Stratégies nutritionnelles pour réduire les agents pathogènes chez la volaille. 5èmes Journ. Rech. Avicole, Tours, France, 31.

Vilarino M., Picard M., Melcion J.P., Faure J.M., 1996. Behavioural adaptation of laying hens to dilution of diets under mash and pellet form. Brit. Poult. Sci., 37, 895-907.

Whitehead C.C., 1998. A review of nutritional and metabolic factors involved in dyschondroplasia in poultry. J. Appl. Anim. Res., 13, 1-16.

Waldenstedt L., 2006. Nutritional factors of importance for optimal leg health in broilers: a review. Anim. Feed Sci. Technol., 126, 291307.

Willemsen H., Debonne M., Swennen Q., Everaert N., Careghi C., Han H., Bruggeman V., Tona K., Decuypere E., 2010. Delay in feed access and spread of hatch: importance of early nutrition. World's Poult. Sci. J., 66, 177-188.

Windish W., Schedle K., Plitzner C., Kroismayr A., 2008. Use of phytogenic products as feed additives for swine and poultry. J. Anim. Sci., 86 (E. Supp.), E140-E148.

Yalcin S., Ozkan S., Turkmut L., Siegel P.B., 2001. Responses to heat stress in commercial and local broiler stocks. 2. Developmental stability of bilateral traits. Brit. Poult. Sci., 42, 149-152.

Yang Y., Iji P.A., Choct M., 2009. Dietary modulation of gut microflora in broiler chickens: a review of the role of six kinds of alternatives to in-feed antibiotics. World's Poult. Sci. J., 65, 97-114.

Yegani M., Korver D.R., 2008. Factors affecting intestinal health in poultry. Poult. Sci., 87, 2052-2063.

\section{Résumé}

La conduite alimentaire peut être impliquée dans l'apparition de troubles locomoteurs, digestifs et métaboliques susceptibles d'altérer le bien-être des poulets de chair. L'objectif de cette revue est de faire le point sur les différentes approches alimentaires et nutritionnelles permettant d'éviter l'apparition de ces désordres et donc d'évaluer la contribution de la gestion de l'alimentation au bienêtre des poulets. Un pré-requis concernant l'alimentation des volailles est que les animaux reçoivent un aliment accessible, sain et équilibré, sachant qu'il n'est pas aisé de définir un besoin nutritionnel appliqué à un troupeau, avec des poulets sélectionnés sur la vitesse de croissance, cette sélection s'accompagnant d'effets qui peuvent paraître délétères vis-à-vis du bien-être animal avec une consommation d'aliment excessive et une faible activité. Différentes solutions alimentaires, parfois antagonistes, sont proposées pour améliorer le bien-être du poulet : adapter la composition et la présentation physique de l'aliment et enfin, jouer sur les modalités de distribution de l'aliment, permettant dans certains cas, d'enrichir leur milieu de vie.

Néanmoins, au regard du bien-être animal, la conduite alimentaire n'est qu'un levier comme le sont la gestion de l'ambiance et le choix du génotype. S'il est clair que l'alimentation s'adapte à l'évolution de la génétique, son effet en matière de bien-être animal semble limité et une réflexion devrait porter sur une amélioration des capacités d'adaptation des poulets. 
Abstract

Managing the feeding process can contribute to broiler chicken welfare

The feeding process can lead to locomotion, digestive and metabolic problems, therefor having an impact on the welfare of poultry. The aim of this study was to investigate different feeding systems in order to avoid these problems and to evaluate if feed management can improve broiler welfare. The first requirement for broiler chickens is to have access to a balanced and healthy diet (feed and water). Since broilers have been selected for high growth rate and feed intake, negative consequences on animal welfare (very high feed intake, poor stimulation for moving) are observed. It is therefore difficult to define an optimal diet.

In the present study, different feeding solutions were reviewed and include changes to feed nutritional composition and physical characteristics after which new feeding systems could also be proposed which could enrich the bird's environment.

Feed management was found to have a limited effect on broiler welfare; whereas other factors such as animal husbandry and genetics are known to have a major role. Selection programmes should take bird adaptation and welfare into account.

MAGNIN M., BOUVAREL I., 2011. Gérer l'alimentation pour contribuer au bien-être des poulets de chair. In : Bien-être du poulet de chair. Dossier, INRA Prod. Anim., 24, 181-190. 